\title{
Trapping of Wide Range Mass-to-Charge Ions and Dependence on Matrix Amount in Internal Source MALDI-FTMS
}

\author{
Arwah J. Jaber, Jacob Kaufman, Rohana Liyanage, Eugenia Akhmetova, \\ Samuel Marney, and Charles L. Wilkins \\ Department of Chemistry and Biochemistry, University of Arkansas, Fayetteville, Arkansas, USA
}

Internal ionization source MALDI-FTMS shows clear variation of number average molecular weight $\left(\mathrm{M}_{\mathrm{n}}\right)$ for an equimolar mixture of four PEG polymers (PEG 2000, PEG 4000, PEG 6000, PEG 8000) when the 2,5-dihydroxy benzoic acid (DHB) matrix to PEG ratio is varied or the laser power is changed. As the matrix to analyte ratio is increased, the analyte signal of higher molecular mass ions increases and the signal of lower mass ions decreases. Laser power dependence studies show a similar trend. Possible explanations for these observations are discussed. (J Am Soc Mass Spectrom 2005, 16, 1772-1780) (C) 2005 American Society for Mass Spectrometry

$\mathrm{M}$ atrix-assisted laser desorption ionization mass spectrometry (MALDI MS) has become a popular technique applied to polymer analysis. Important information on polymer structure, composition, and molecular weight can be generated directly from the MALDI spectra [1-4]. Determination of polymer molecular weight distributions is a valuable tool for understanding the relationships of those distributions and polymer properties.

A generally challenging problem in MALDI Fourier transform mass spectrometry (FTMS) application to polymer characterization is determination of the actual molecular weight distribution, especially when analyzing polymers possessing a high degree of polydispersity. The difficulty in efficiently trapping ions over a wide range of mass-to-charge ratio becomes apparent when high mass ions are involved. A variety of experimental and theoretical approaches have been reported to improve the trapping efficiencies of high mass ions $[5,6]$. Fourier transform mass spectrometry employing a gated trapping deceleration approach was first applied by Wilkins and coworkers and achieved muchimproved results for low and high mass ions [7-9].

Detector mass discrimination is another problem when dealing with polydisperse polymers. If the detector responds differentially to ions, preferentially favoring detection of ions of one mass versus those of another, the phenomenon is called mass discrimination. Arising from several factors, [10-18] mass discrimination has also been reported in the analysis of proteins,

Published online September 22, 2005

Address reprint requests to Dr. C. L. Wilkins, Department of Chemistry and Biochemistry, University of Arkansas, Fayetteville, AR 72701, USA. E-mail: cwilkins@comp.uark.edu narrow molecular weight polymer distributions, and uniform oligomer mixtures.

Previously, Dey et al. [10] reported mass discrimination in MALDI-FTMS when gated deceleration times were systematically varied. It was pointed out that the use of a single deceleration time can lead to inaccurate molecular weight determination. Therefore, these workers advocated an averaging approach. A unifying theme among mass discrimination studies is the fact that one must pay careful attention to instrument parameters and to sample preparation for accurate polymer distribution determination to result. In the present study, enhanced signals are observed for high mass ions when an equimolar four-component polyethylene glycol blend is analyzed with increased matrix-to-analyte molar ratio. Thus, the apparent number average molar mass $\left(\mathrm{M}_{\mathrm{n}}\right)$ of the mixture is affected by the amount of matrix added. Experimentally, it is found that proper choice of matrix-to-analyte molar ratio is critical in optimizing the FTMS signal for observation of a wide mass range of ions. Accordingly, possible explanations for these observations are suggested.

\section{Experimental}

\section{Materials and Sample Preparation}

Polymer samples of PEG 2000, PEG 4000, PEG 6000, PEG 8000, and the matrix 2,5-dihydroxybenzoic acid (DHB) were obtained from Fluka (Buchs, Switzerland) and used as received. 2-(4-Hydroxyphenylazo)-benzene acid (HABA) was obtained from Aldrich (St. Louis, $\mathrm{MO}$ ). Methanol was obtained from EM Science (Norwood, $\mathrm{OH}$ ). Tetrahydrofuran (THF) was obtained from 

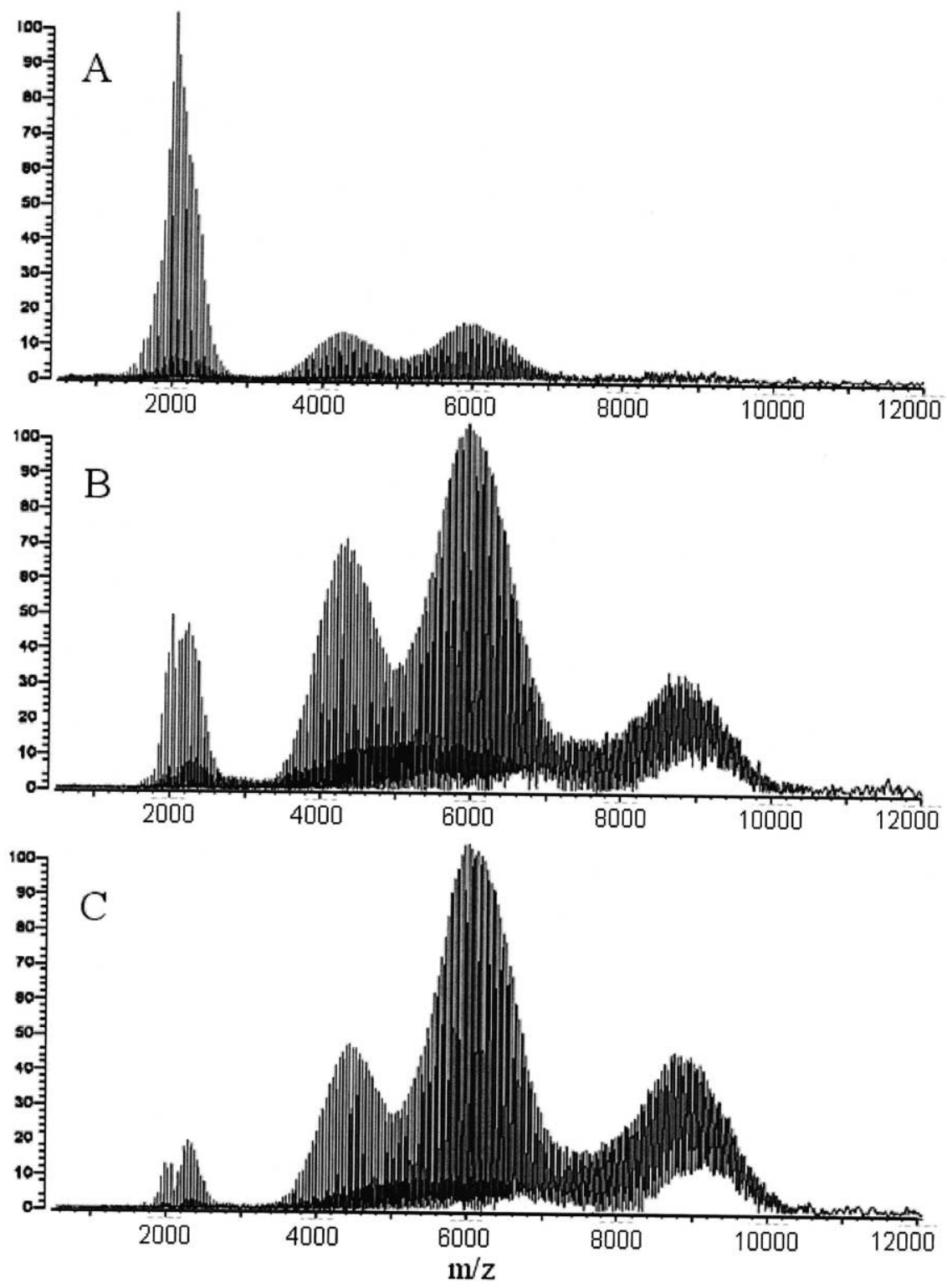

Figure 1. Signal intensity dependence on the matrix-to-analyte (M/A) molar ratio at constant deceleration time under same laser fluence. Spectrum A has matrix-to-analyte ratio of 800:1:1:1:1 DHB/PEG, Spectrum B has matrix-to-analyte ration of 4000:1:1:1:1, and Spectrum C has matrix-toanalyte ratio $60,000: 1: 1: 1: 1$.

J. T. Baker (Phillipsburg, NJ). All reagents were used without further purification.

An equimolar $(0.005 \mathrm{M})$ mixture solution containing $2,4,6$, and $8 \mathrm{~K}$ PEG samples was prepared in methanol. For sample preparation, these samples together with a 1.0 M solution of DHB matrix in methanol were used. The HABA matrix was dissolved in THF to achieve a concentration of $0.5 \mathrm{M}$. The analytes were mixed with respective matrices to give the following matrix-toanalyte (M/A) molar ratios (matrix:PEG2K:PEG4K: PEG6K:PEG8K) 400:1:1:1:1， 800:1:1:1:1， 1200:1:1:1:1, 2000:1:1:1:1， 3200:1:1:1:1， 4000:1:1:1:1， 20,000:1:1:1:1， 40,000:1:1:1:1, 60,000:1:1:1:1, 80,000:1:1:1:1, and 100,000: 1:1:1:1. All samples were aerosprayed onto stainless steel probe tips in the same way to ensure homogeneity of the samples. The aerospray method has been described elsewhere [9].

\section{Instrumentation}

MALDI experiments were performed using a 7.2 tesla (Oxford Instrument, Clearwater, FL) Fourier transform mass spectrometer equipped with an internal ionization source. The FTMS instrument was retrofitted with an IonSpec data acquisition station (Irvine, CA) that was employed for experiment control and data analysis. A pulsed Nd-YAG laser operating at a wavelength of $355 \mathrm{~nm}$ was used for MALDI. All 


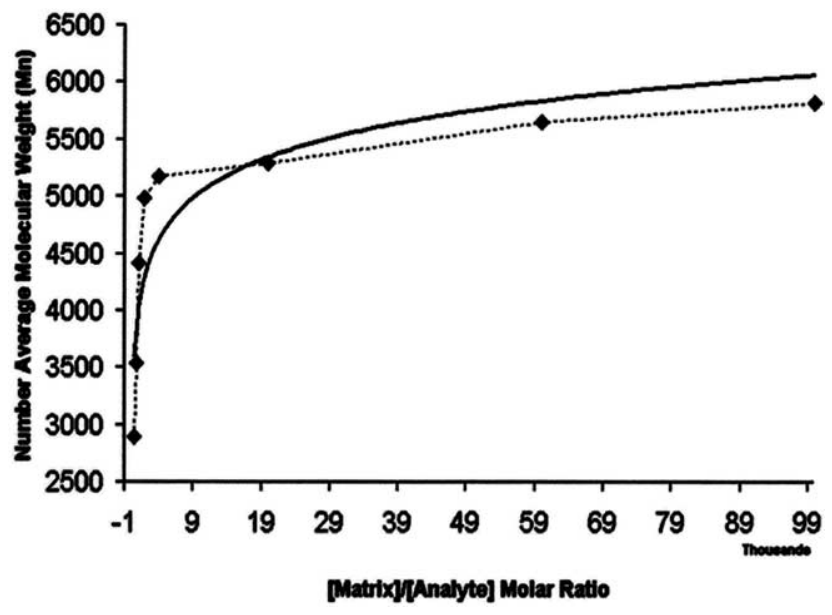

Figure 2. An illustration of increase in the number average molecular weight $\left(M_{n}\right)$, due to more efficient trapping of high mass molecular ions, as the amount of matrix added is increased. The dashed line is for eye guidance and the solid line is a logarithmic fit through the data points (the -1 shown in scale was added to separate the point from the y-axis). In this Figure and in Figure 6, number of average molecular weight was calculated as $M_{n}=\Sigma M_{i} R_{i} / \Sigma R_{i}$, where $M_{i}$ is the mass and $R_{i}$ the relative abundance.

MALDI-FTMS spectra were acquired in positive ion mode at a pressure of $\sim 5 \times 10^{-7}$ torr. The source and analyzer cells of the cubic dual cell are separated by a conductance limit with a 2-mm diameter hole. The vacuum chamber was pumped by two diffusion pumps (Edwards Diffstak Model 160, Wilmington,
MA), each with a maximum pumping speed of 700 $\mathrm{L} / \mathrm{s}$. For the HABA matrix results, cryogenic pumps replaced the diffusion pumps (Austin Scientific, Cryoplex 8, Austin, TX) and have maximum pumping speed of $1500 \mathrm{~L} / \mathrm{s}$. Samples were desorbed from a stainless steel probe tip of an automated solids probe, situated $2 \mathrm{~mm}$ from the front trapping plate of the source analyzer cell. Ions were trapped and analyzed in the 17/8-inch cubic source cell region of the dual cubic cells. Each of the dual cubic source cells is composed of two parallel receiver plates, two transmitter plates, a front plate, and a rear trapping plate (the conductance limit of the dual cell is situated in the trap plate separating the two cells). To trap positive ions, the front trapping plate is set at $0.0 \mathrm{~V}$, and the rear trapping plate is set between 9.0 and 9.5 $\mathrm{V}$ during the ionization event. The decelerating potential is maintained for a variable time of between 125-500 $\mu$ s after firing the laser. Subsequently, the potentials of the front and the rear plates are adjusted to 2.0 trapping voltage. Once ions are trapped, a 125 ms delay is imposed so that the laser-desorbed neutrals can be pumped away and analyte ions permitted to relax to the center of the cell and equilibrate within the trap. For mass spectral acquisition, ions are excited for $0.5 \mathrm{~ms}$ by applying a $200 \mathrm{~V}$ peak-to-peak frequency sweep with a general range of $10-220 \mathrm{KHz}$ and a sweep rate of $420 \mathrm{~Hz} / \mu \mathrm{s}$. All detection was done in direct mode and $128 \mathrm{~K}$ data points were collected at the rate of $2 \mathrm{Mhz}$. Each spectrum is the sum of 50 individual transients. Each transient results

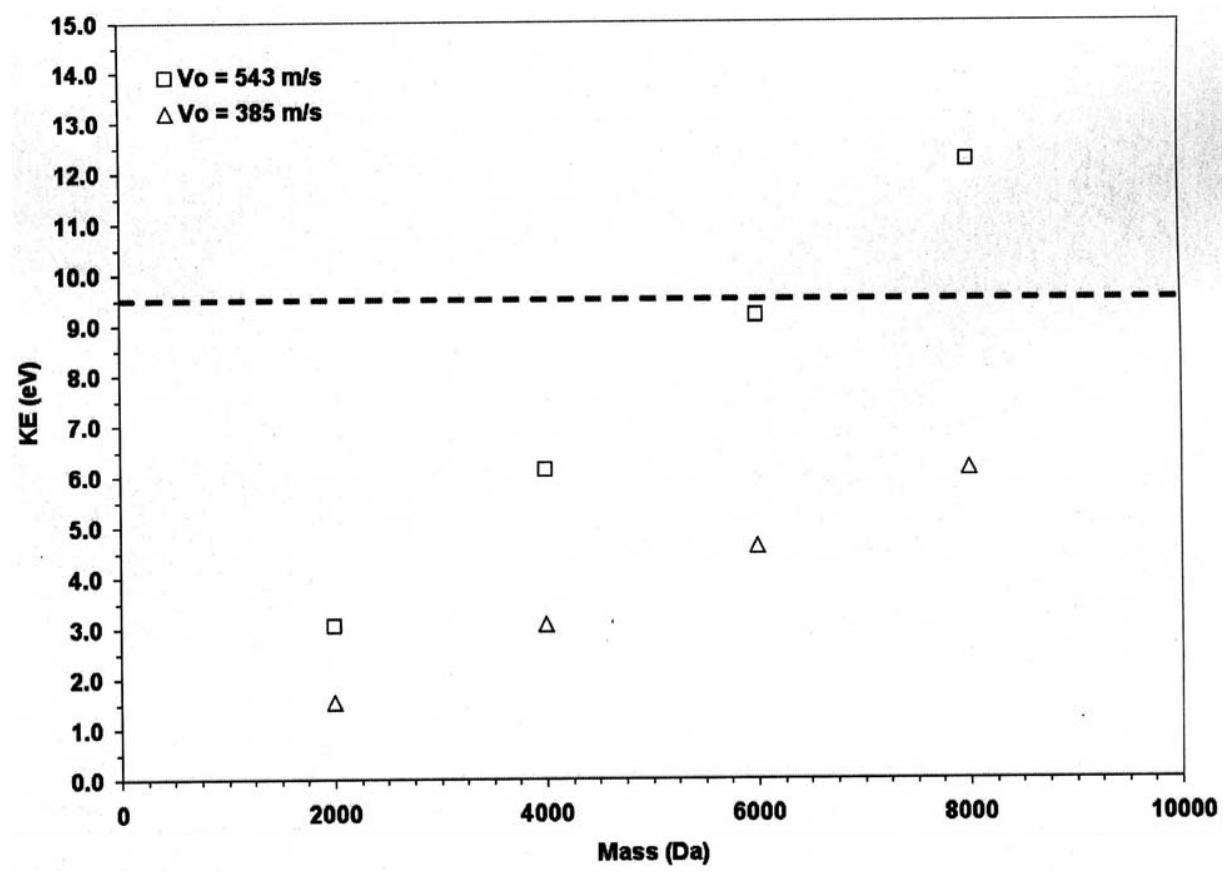

Figure 3. Considering the masses 2000, 4000, 6000, and $8000 \mathrm{Da}$, their kinetic energy in electron volt units is plotted as a function of selected initial ion velocities. Ions above the dashed horizontal line are not decelerated efficiently and those below the line will be lost. Kinetic energy was calculated as KE $=\mathrm{mv}^{2} / 2$. 

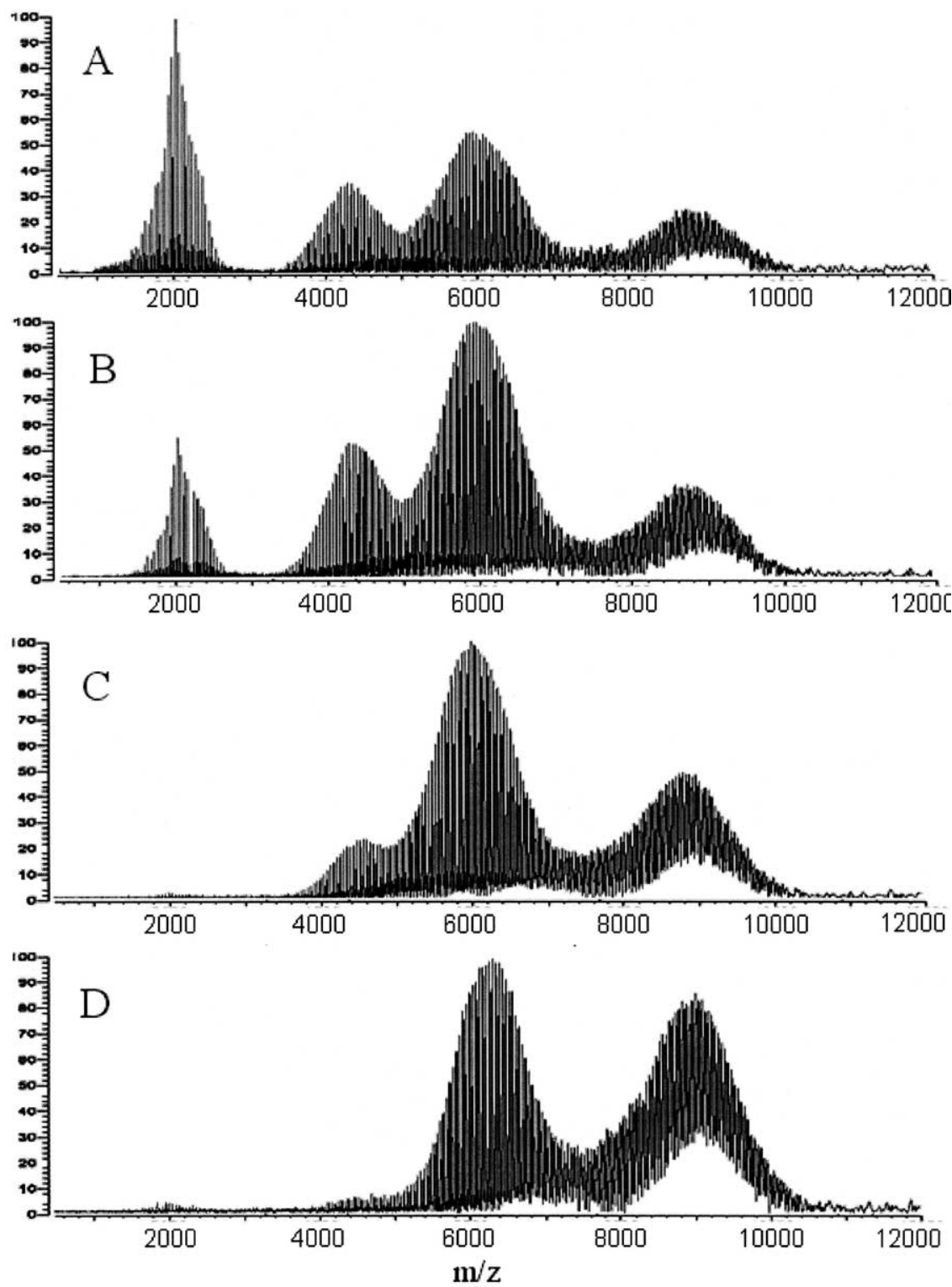

Figure 4. Ion abundance as a function of increasing deceleration times. Spectrum $\mathrm{A}=0.125 \mathrm{~ms}$, Spectrum B $=0.225 \mathrm{~ms}$, Spectrum C $=0.325 \mathrm{~ms}$, Spectrum D $=0.425 \mathrm{~ms}$. The matrix-to-analyte molar ratio is $20,000: 1: 1: 1: 1$.

from a single laser pulse at the same spot on the MALDI target. All spectra were plotted in relative abundance scale on the y-axis, and when absolute scale was used no difference was observed.

\section{Results and Discussion}

It would be desirable for polymer blend formulation to monitor the mixture by mass spectrometry. Ideally, for a polymer blend comprised of an equimolar mixture of four PEG polymers, as examined here, with mass spectrometry one should observe four distributions of the individual PEG polymer mixtures. PEG ions resulting from these distributions should be expected to have the same ionization efficiency and would logically be de- tected in similar abundance. However, in practice it is difficult to obtain such a result due to the influence of various factors such as matrix concentration, [19-21] sample morphology, [22] crystallite size, [23] buffer type, [24, 25] laser energy, [26] delayed extraction, [27] cation choice, [28, 29], and laser fluence [30-32]. Figure 1 contains MALDI-FTMS spectra obtained for an equimolar mixture of PEG $2 \mathrm{~K}, 4 \mathrm{~K}, 6 \mathrm{~K}$, and $8 \mathrm{~K}$ as a function of relative amount of DHB matrix added. This figure shows the mass spectra obtained for the matrixto-analyte molar ratios of 800:1:1:1:1, 4000:1:1:1:1, and 60,000:1:1:1:1. All experimental conditions except the matrix-to-analyte molar ratio (M/A), were kept constant. It is obvious that there are dramatic differences between these spectra and that, as the matrix ratio is 

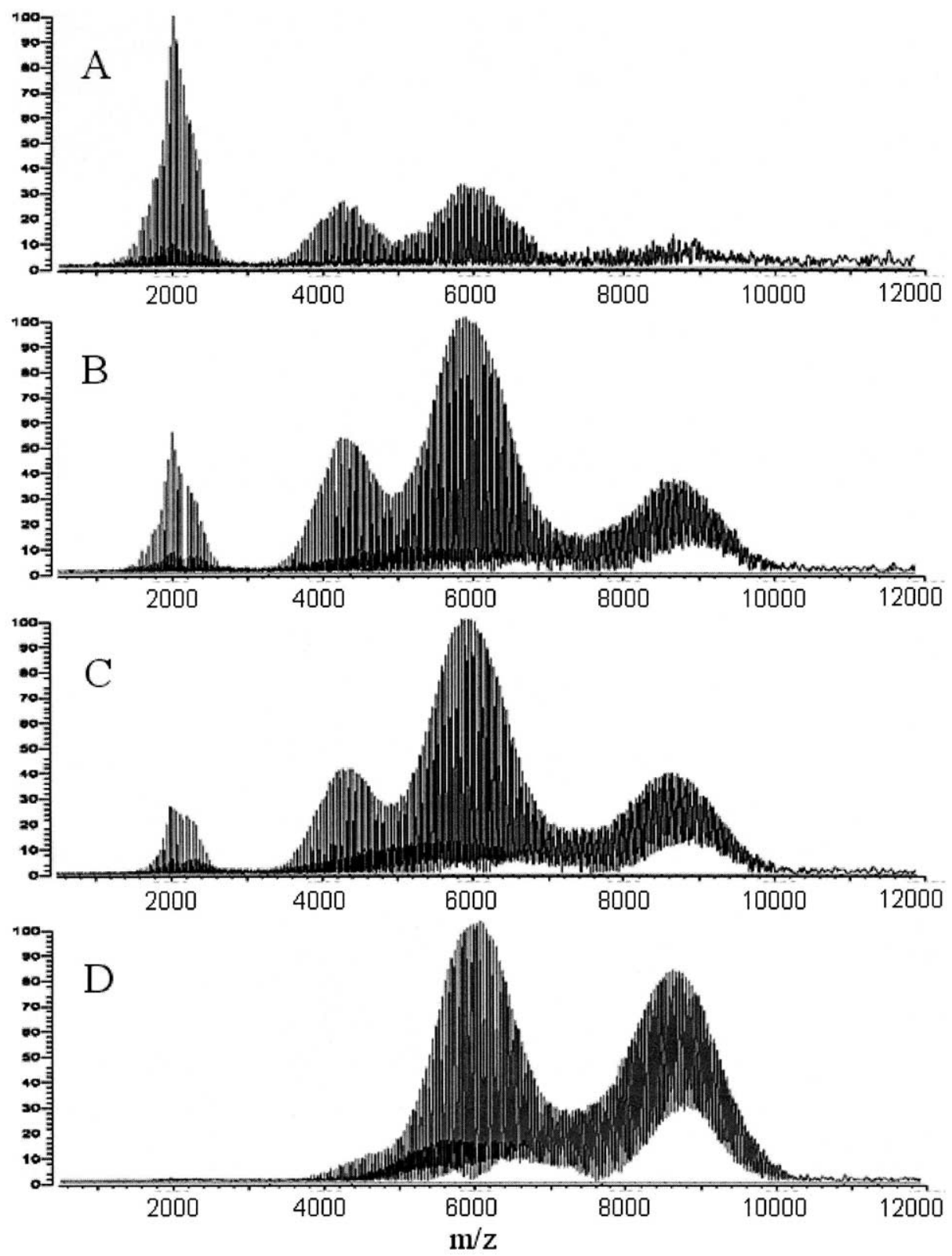

Figure 5. Dependence of ion abundance on the influence of laser energy. Spectrum $A=0.79 \mathrm{~mJ}$, Spectrum B $=0.93 \mathrm{~mJ}$, Spectrum C $=1.08 \mathrm{~mJ}$, Spectrum $\mathrm{D}=1.35 \mathrm{~mJ}$. The matrix-to-analyte molar ratio is $20,000: 1: 1: 1: 1$. The trapping time was $125 \mathrm{~ms}$; the retarding potential was $9.5 \mathrm{~V}$; the trapping potential was $2.0 \mathrm{~V}$ on both the front and back plate.

increased, the abundances of the higher mass ions have grown at the expense of those of lower mass. The correlation can be clearly seen in Figure 2. As the M/A ratio is increased, an increase in the apparent $M_{n}$ value is observed. This trend fits a logarithmic scale as illustrated by the solid line. These data raise interesting questions. Why is the relative signal of high mass ions improved as a function of matrix concentration? What effect does the M/A ratio have on the kinetic energy of desorbed ions of low and high molecular weight?

Molecular dynamics calculations frequently have been employed to describe MALDI. One theory suggests that cluster formation might play an important role, offering a possible interpretation of the present results [33-35]. Computer simulations indicate that clusters and chunks of ions are ejected during MALDI event with varying velocities, sizes, and rates of decay. The velocity distributions of desorbed MALDI ions have been the subject of several papers [34, 36-40]. Models have also emerged to address the mechanism of desorbed MALDI ions and have focused on two processes: molecular jet expansion [39] and cluster-like expansion [33, 35, 41, 42]. The jet model, as put forward by Beavis and Chait [39], proposes that analytes are entrained in a jet of matrix, causing them to assume the velocity of the matrix independent of analyte mass. In 


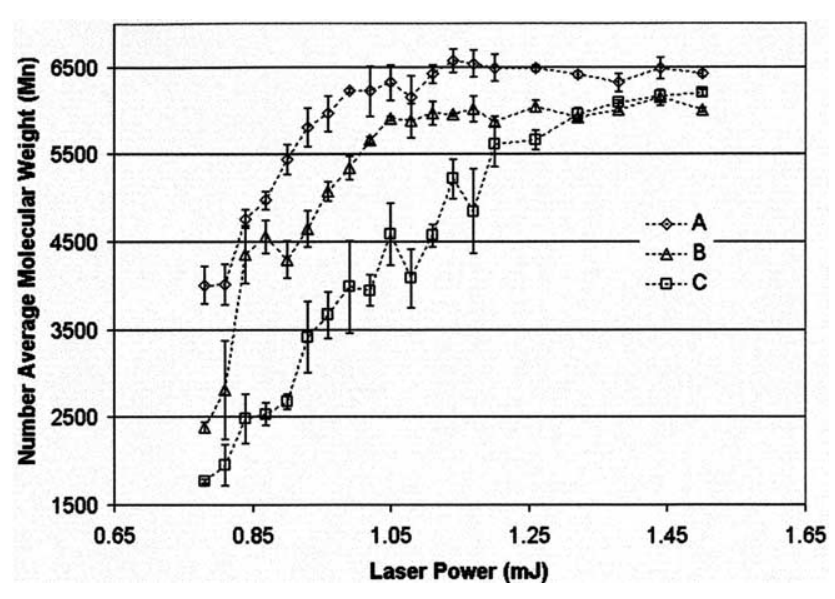

Figure 6. Examples of the shift in the $M_{n}$ value caused by the influence of laser power. The matrix-to-analyte molar ratios are 40,000:1:1:1:1, 3200:1:1:1:1, and 800:1:1:1:1 in A, B, and C, respectively. The dashed lines are for eye guidance.

contrast, the cluster model suggests a decay mechanism for analyte clusters and that initial velocities decrease as the masses of the analytes increase [34]. Furthermore, Gluckmann and Karas [36] have reported some important features of the ion initial velocity distributions. They found that the initial ion velocities of peptides and proteins for a given matrix were independent of their mass to charge ratio, but matrix-dependent ranging from $273-543 \mathrm{~m} / \mathrm{s}$ for the matrices studied. However, depending on the nature of the mixed additive and analytes to the DHB matrix, Gluckmann and Karas [36] found significant changes in the initial velocities of ions. Thus, it was noted that initial velocity decreased when DHB was desorbed with carbohydrates compared with the original initial ion velocity from a pure DHB matrix.

The fact that the energy of MALDI-generated ions is a function of ion mass (i.e., the kinetic energy increases with increase in mass) is important for trapping and detection of ions in MALDI-FTMS and should apply to all ions desorbed from a DHB matrix when different relative concentrations of matrix and analytes are added. For this reason, it is postulated that increasing the relative amount of matrix may contribute to a change in the mean kinetic energy of desorbed ions. The question is what effects do kinetic energy changes have on velocities of analytes within a matrix plume upon expansion? For example, the initial ion velocity of insulin desorbed from pure DHB matrix has been reported to be $543 \mathrm{~m} / \mathrm{s}( \pm 40$ $\mathrm{m} / \mathrm{s}$ ) [36], and if this velocity is assumed to be the same for polymers, Figure 3 suggests that ions with kinetic energy below the horizontal line at $9.5 \mathrm{~V}$ can be decelerated successfully, mainly masses up to $\mathrm{m} / \mathrm{z}$ 6000. If the velocity of the DHB is assumed to be as low as $\sim 385 \mathrm{~m} / \mathrm{s}$, single-charged ions with masses up to 8000 Da can be decelerated effectively if a retarding potential is $9.5 \mathrm{eV}$ is used. Therefore, for ion deceleration, any strategy that reduces the initial matrix velocity would be advantageous. A decrease in M/A should lead to this result. Similar decreases are observed when DHB is mixed with maltotetraoses and chitotetraoses [36]. In cases where the $\mathrm{M} / \mathrm{A}$ ratio approaches the composition of pure DHB, it is logical to assume that the velocity of the desorbed polymer ions to be closer to $543 \mathrm{~m} / \mathrm{s}$.

Although all PEG ions emerge from the same sample spot, some significant differences can be inferred from the different matrix loadings. It is reasonable to propose that the amount of matrix added has an effect on the kinetic energy of the analyte ions. After ions are desorbed from the MALDI target, they emerge with a certain kinetic energy and move along the z-axis of the magnetic field. This kinetic energy is decreased because of the influence of the decelerating potential. Kinetic energy is then converted into potential energy. If a decelerating potential higher than the kinetic energy of the ions is applied, the ions will be repelled, and eventually turn around and be accelerated in the opposite direction. The higher the initial kinetic energy, the longer deceleration takes. Higher mass ions will be decelerated more slowly than lower mass ions. Therefore, which ions are trapped depends precisely on deceleration time and when the trapping voltages are applied (+2 V).

As a confirmation of this theory [10], the deceleration time was systematically varied, producing the results in Figure 4. At a constant decelerating potential energy applied to the rear trap plate, low mass ions were trapped at lower deceleration times, while larger ones were not trapped efficiently because they were decelerated too slowly (i.e., more of them escaped before being decelerated to the $2 \mathrm{~V}$ trapping voltage). Conversely, at longer deceleration times, higher mass ions were more efficiently trapped than lower mass ions because preferential loss of lower mass ions occurred.

To substantiate that the matrix has affected the velocities of the desorbed ions, a series of experiments were carried out in which the laser power was systematically raised while keeping all other parameters unchanged. It was reasoned that the influence of raising the desorption laser energy would increase the velocity of desorbed ions. If this is so, then the trappable number of ions will change as a function of raising the laser energy. The maximum laser output is $3.0 \mathrm{~mJ}$ with a threshold of $0.70 \mathrm{~mJ}$ for the onset of spectral signal. Figure 5 summarizes the laser power dependence studies performed for PEG $2 \mathrm{~K}, 4 \mathrm{~K}, 6 \mathrm{~K}$, and $8 \mathrm{~K}$. The M/A molar ratio and all the other experimental conditions were kept constant. In Spectrum A of Figure 5 the laser power was set at about $0.79 \mathrm{~mJ}$, just above the threshold. In Spectrum D of Figure 5 the power was $1.35 \mathrm{~mJ}$. Going beyond $1.5 \mathrm{~mJ}$, the spectra resembled that of the Figure $5 \mathrm{~d}$ and resulted in ablating the sample quickly from the surface (basically the sample is depleted). Similar to what was observed for M/A ratio studies, mass discrimination can result from an increase in laser power as is evident from the calculated $M_{n}$ values shown in Figure 6. The three examples shown in this 

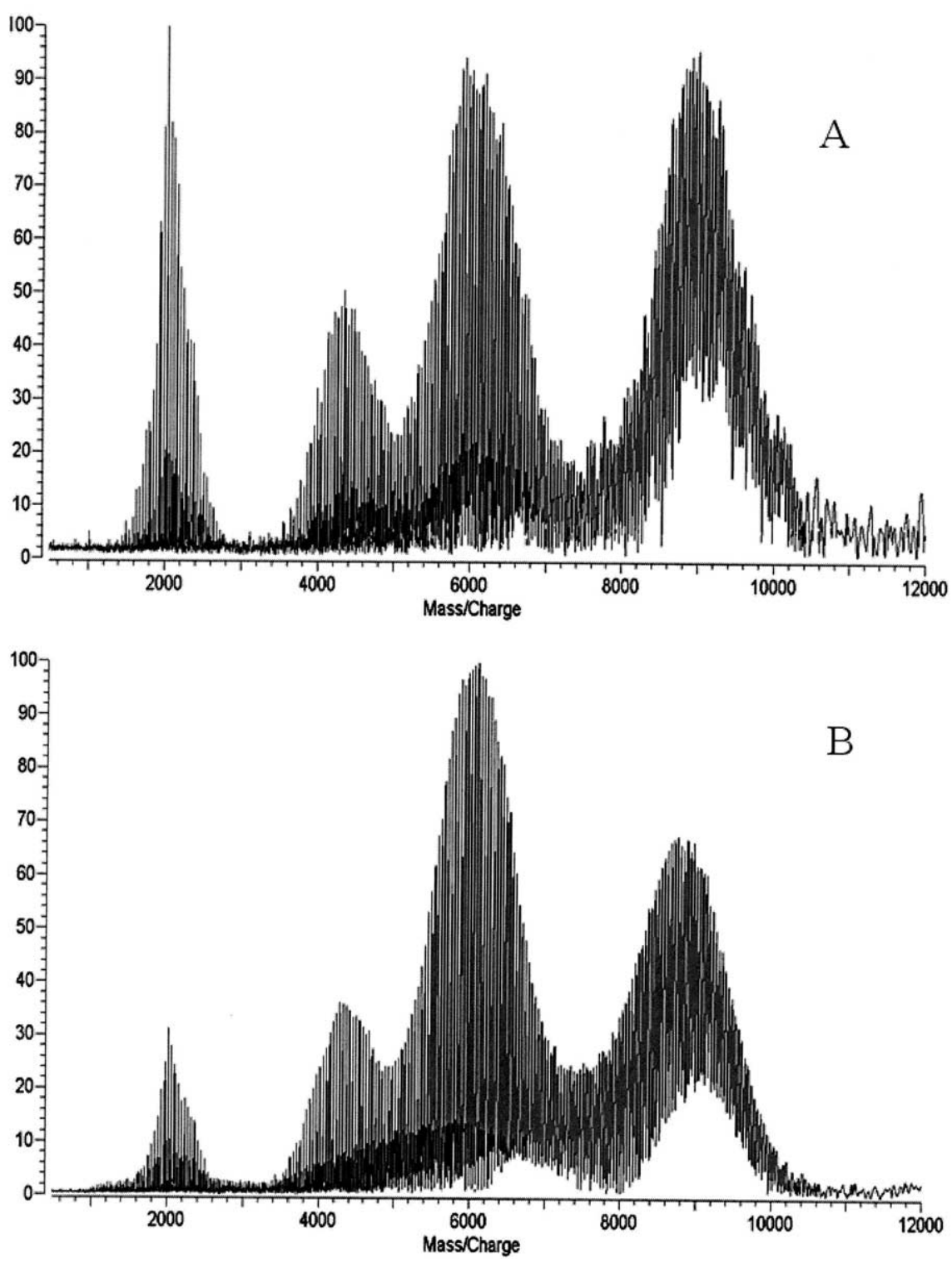

Figure 7. Comparison between the observed signal from same sample with same M/A ratio, and same other instrumental setup but different matrix. Spectrum A was acquired with HABA matrix. Spectrum B was obtained with DHB matrix.

figure share one thing in common, that is, they all show an increase in the $M_{n}$ value up to a limit at mass $\sim 6500$, where the lines start flattening. This flattening limit occurs because at highest laser power, only the $6 \mathrm{~K}$ and the $8 \mathrm{~K}$ distributions are observed, with $6 \mathrm{~K}$ distribution having equal or higher intensity than the $8 \mathrm{~K}$ PEG sample, and so, when the number average molecular Weight $\left(M_{n}\right)$ formula is applied, a value of about 6500 for the average mass is obtained.

Again, the trends shown in Figure 6 can be explained as a result of initial ion velocity change of PEG molecules with the laser power. Possibly, when the amount of energy deposited on the MALDI surface increases, sample temperature and thermal vaporization increases [38]. Consequently, the ther- mal motion of ejected molecules would increase under higher laser power [43].

Low molecular weight ions are preferentially detected at low laser power, while at higher laser power; larger ions are detected in greater abundance. Detection of ions in an internal source FTMS instrument must occur after trapping ions in an electrostatic potential well, so that the trapping efficiency is closely associated with the initial ion velocity. Thus, the FTMS mass discrimination observed can be explained by dependence of ion axial velocities on laser energy and by changes in matrix composition. This premise is in accord with previously reported experimental and theoretical observations that the average initial ion velocities increase with higher laser fluence [34,35]. It can be 
seen from the present results that other factors besides laser power affecting the velocity of ions produced by MALDI may also result in mass discrimination effects in spectra obtained using internal FTMS sources.

Figure 7 compares two spectra obtained using different matrices but with other parameters held constant. These spectra show that with the HABA matrix, relatively efficient trapping of all ions is observed. However, for the DHB matrix the observed signal favors higher mass. It was reported in reference [24] that the insulin had a velocity of $273 \mathrm{~m} / \mathrm{s}$ when it was desorbed from the HABA matrix in comparison with $543 \mathrm{~m} / \mathrm{s}$ when it is desorbed from the DHB matrix. Although the exact velocity of the polymer ions in the present measurement cannot be determined by this instrumental setup, the results allow inference of whether the ions were traveling at a slow or fast rate. Therefore, it is seen that when using HABA matrix which normally produces low velocity ions, different spectral results are obtained compared to those with DHB. Interestingly, and in accord with the explanations offered above, for HABA no matrix ratio dependence was observed when different $\mathrm{M} / \mathrm{A}$ ratios were examined. This observation is expected because in this case all PEG ions will have both low initial velocities and kinetic energies. Hence, at a given time during the deceleration period, all will have lower kinetic energy than at the corresponding time for the DHB case. Furthermore, these lower kinetic energy ions will undergo smaller kinetic energy changes as a result of variations of $\mathrm{M} / \mathrm{A}$.

It is also worth noting that the MALDI-TOF spectra were the same for various $\mathrm{M} / \mathrm{A}$ choices. On varying M/A of 800:1 to M/A 60,000:1 ratios for both HABA and $\mathrm{DHB}$, the spectra were qualitatively unchanged. The observed spectral signal for the four distributions was in the following order: PEG $2 \mathrm{k}$ had the highest apparent abundances followed by the $4 \mathrm{~K}, 6 \mathrm{~K}$, and $8 \mathrm{~K}$ which had the lowest intensity. This is because all ions were extracted from the source and accelerated to approximately the same KE, and are therefore separated primarily based upon their masses.

\section{Conclusions}

Efficient trapping of wide range mass-to-charge ions may depend on various factors. In the present case, employing an internal source MALDI-FTMS system, the results are greatly influenced by the matrix/analyte ratio chosen. This can be caused by the matrice's effect upon ion velocities. It is not possible to exactly pinpoint the mechanism by which the ion plume expands. From the present data, it is speculated that both mechanisms are involved; ions are expanding in both with cluster and jet-like mechanisms, consistent with literature results [44], [34].öThus,öwhenötheömatrixöisöpresentöin lesser amounts, the velocity of the desorbed species tends to be slower, and when the matrix is present in higher amount, the velocity of the analytes becomes closer to that of the matrix. Because of the influence of the deceleration voltage, low mass ion velocities increase and high mass velocities decrease by the time the trapping voltage $(+2 \mathrm{~V})$ is applied, resulting in mass discrimination. Unfortunately, the signal intensity does not scale linearly with a particular matrix-to-analyte ratio; thus it is difficult to predict the exact amount of matrix required. Laser energy also plays a significant role in desorption/ionization of larger molecules. Therefore, use of optimum laser power will facilitate and contribute to accurate mass measurements.

\section{Acknowledgments}

The authors gratefully acknowledge support from National Science Foundation grants CHE-00-91,868, CHE-99-82,045, and CHE04-55,134. They also thank Mr. Jerry Homesley for his valuable assistance in repairing the 7.2 tesla instrument and Mr. Jeff Jones for helpful discussions.

\section{References}

1. Hanton, S. D. Mass Spectrometry of Polymers and Polymers Surfaces. Chem. Rev. 2001, 101, 527-569.

2. Karas, M.; Bahr, U.; Ingendoh, A.; Hillenkamp, F. Laserdesorption Mass Spectrometry of 100,000-250,000-Dalton Proteins. Angew. Chem. 1989, 101, 805-806.

3. Nielen, M. W. F. MALDI Time-of-Flight Mass Specrometry of Synthetic Polymers. Mass Spectrom. Rev. 1999, 18, 309-344.

4. Rader, H. J.; Schrepp, W. MALDI-TOF Mass Spectrometry in the Analysis of Synthetic Polymers. Acta Polym. 1998, 49, 272-293.

5. Remple, D. L.; Gross, M. L. A Gated Trapping Strategy with Two Time Constants and a Delay to Trap Ions Over a Broad Range of Mass-to-Charge and Velocity. J. Am. Soc. Mass Spectrom. 2001, 12, 296.

6. Frankevich, V.; Zenobi, R. Deceleration of High-Energy Matrix-Assisted Laser Desorption/Ionization Ions in an Open Cell for Fourier Transform Ion Cyclotron Resonance Mass Spectrometry. Rapid Commun. Mass Spectrom. 2001, 15, 20352040.

7. Castoro, J. A.; Wilkins, C. L. Ultrahigh Resolution MatrixAssisted Laser Desorption Ionization of Small Proteins by Fourier Transform Mass Spectrometry. Anal. Chem. 1993, 65, 2621-2627.

8. Köster, C.; Castoro, J. A.; Wilkins, C. L. High Resolution Matrix-Assisted Laser Desorption/Ionization of Biomolecules by Fourier Transform Mass Spectrometry. J. Am. Chem. Soc. 1992, 114, 7572-7574.

9. Yao, J.; Dey, M.; Pastor, S. J.; Wilkins, C. L. Analysis of High-Mass Biomolecules Using Electrostatic Fields and Matrix-Assisted Laser Desorption/Ionization in a Fourier Transform Mass Spectrometer. Anal. Chem. 1995, 67, $3638-$ 3642.

10. Dey, M.; Castoro, J. A.; Wilkins, C. L. Determination of Molecular Weight Distributions of Polymers by MALDIFTMS. Anal. Chem. 1995, 67, 1575-1579.

11. Farmer, T. B.; Caprioli, R. M. Mass Discrimination in MatrixAssisted Laser Desorption Ionization Time-of-Flight Mass Spectrometry: A Study Using Cross-Linked Oligomeric Complexes. J. Mass Spectrom. 1995, 30, 1245-1254.

12. Martin, K.; Spickermann, J.; Rader, H. J.; Mullen, K. Why Does Matrix-Assisted Laser Desorption/Ionization Time-of-Flight Mass Spectrometry Give Incorrect Results for Broad Polymer 
Distributions? Rapid Commun. Mass Spectrom. 1996, 10, 1471-1474.

13. Shimada, K.; Lusenkova, M. A.; Sato, K.; Saito, T.; Matsuyama, S.; Nakahara, H.; Kinugasa, S. Evaluation of Mass Discrimination Effects in the Qualitative Analysis of Polydisperse Polymers by Matrix-Assisted Laser Desorption/Ionization Timeof-Flight Mass Spectrometry Using Uniform Oligostyrenes. Rapid Commun. Mass Spectrom. 2001, 15, 277-282.

14. Schriemer, D. C.; Li, L. Mass Discrimination in the Analysis of Polydisperse Polymers by MALDI Time-of-Flight Mass Spectrometry. 2. Instrumental Issues. Anal. Chem. 1997, 69, 41764183.

15. Chen, H.; Guo, B. Use of Binary Solvent Systems in the MALDI-TOF Analysis of Poly(methyl methacrylate). Anal. Chem. 1997, 69, 4399-4404.

16. Rashidzadeh, H.; Guo, B. Use of MALDI-TOF to Measure Molecular Weight Distributions of Polydisperse Poly(methyl methacrylate). Anal. Chem. 1998, 70, 131-135.

17. Belov, M. E.; Nikolaev, E. N.; Harkewicz, R.; Masselon, C. D.; Alving, K.; Smith, R. D. Ion Discrimination During Ion Accumulation in a Quadrupole Interface External to a Fourier Transform Ion Cyclotron Resonance Mass Spectrometer. Int. J. Mass Spectrom. 2001, 208, 205-225.

18. Van Rooij, G. J.; Boon, J. J.; Duursma, M. C.; Heeren, R. M. A. Probing Mass Discrimination and Mass Shifts in the ITMS Mass Spectra of Externally Generated MALDI Ions with Synthetic Polymers. Int. J. Mass Spectrom. 2002, 221, 191-207.

19. Chan, D. T. W.; Colburn, A. W.; Derrick, P. J. Matrix-Assisted UV Laser Desorption. Suppression of the Matrix Peaks. Org. Mass Spectrom. 1991, 26, 342-344.

20. Chan, D. T. W.; Colburn, A. W.; Derrick, P. J.; Gardinar, D. J.; Bowden, M. Suppression of Matrix Ions in Ultraviolet Laser Desorption: Scanning Electron Microscopy and Raman Spectroscopy of the Solid Samples. Org. Mass Spectrom. 1992, 27, 188-194.

21. Knochenmuss, R.; Karbach, V.; Wiesli, U.; Breuker, K.; Zenobi, R. The Matrix Suppression Effect in Matrix-Assisted Laser Desorption/Ionization: Application to Negative Ions and Further Characteristics. Rapid Commun. Mass Spectrom. 1998, 12, 529-534.

22. Gusev, A. I.; Wilkinson, W. R.; Proctor, A.; Hercules, D. M. Improvement of Signal reproducibility and Matrix/Comatrix Effects in MALDI Analysis. Anal. Chem. 1995, 67, 1034-1041.

23. Sadeghi, M.; Vertes, A. Crystallite Size Dependence of Volatilization in Matrix-Assisted Laser Desorption Ionization. Appl. Surf. Sci. 1998, 127/129, 226-234.

24. Kallweit, U.; Bornsen, K. O.; Kresbach, G. M.; Widmer, H. M. Matrix Compatible Buffers for Analysis of Proteins with Matrix-Assisted Laser Desorption/Ionization Mass Spectrometry. Rapid Commun. Mass Spectrom. 1996, 10, 845-849.

25. Yao, J.; Scott, J. R.; Young, M. K.; Wilkins, C. L. Importance of Matrix:Analyte Ratio for Buffer Tolerance Using 2,5Dihydroxybenzoic Acid as a Matrix in Matrix-Assisted Laser Desorption/Ionization-Fourier Transform Mass Spectrometry and Matrix-Assisted Laser Desorption/Ionization-Time of Flight. J. Am. Soc. Mass Spectrom. 1998, 9, 805-813.

26. Knochenmuss, R.; Dubois, F.; Dale, M. J.; Zenobi, R. The Matrix Suppression Effect and Ionization Mechanisms in Matrix-Assisted Laser Desorption/Ionization. Rapid Commun. Mass Spectrom. 1996, 10, 871-877.

27. Mowat, I. A.; Donovan, R. J.; Maier, R. R. J. Enhanced Cationization of Polymers Using Delayed Ion Extraction with Matrix-Assisted Laser Desorption/Ionization. Rapid Commun. Mass Spectrom. 1997, 11, 89-98.
28. Hoberg, A.-M.; Haddleton, D. M.; Derrick, P. J.; Jackson, A. J.; Scrivens, J. H. The Effect of Counter Ions in Matrix-Assisted Laser Desorption/Ionization of Poly(methyl methacrylate). Eur. Mass Spectrom. 1998, 4, 435-440.

29. Okuno, S.; Ohmoto, M.; Arakawa, R. Analysis of Polypropyleneglycodes using Electrospray Ionization Mass Spectrometry. Effects of Cationizing Agents on the Mass Spectra. Eur. J. Mass Spectrom. 2003, 9, 97-103.

30. Feldhaus, D.; Menzel, C.; Berkenkamp, S.; Hillenkamp, F.; Dreisewerd, K. Influence of Laser Fluence in Infrared MatrixAssisted Laser Desorption/Ionization with 2.94 um Er:YAG Laser and Flat-Top Beam Profile. J. Mass Spectrom. 2000, 35, $1320-1328$.

31. Gong, W.; Elitzin, V. I.; Janardhanam, S.; Wilkins, C. L.; Fritsch, I. Effect of Laser Fluence on Laser Desorption Mass Spectra of Organothiol Self-Assembled Monolayers on Gold. J. Am. Chem. Soc. 2001, 123, 769-770.

32. Westmacott, G.; Ens, W.; Hillenkamp, F.; Dreisewerd, K.; Schurenberg, M. The Influence of Laser Fluence on Ion Yield in Matrix-Assisted Laser Desorption Ionization Mass Spectrometry. Int. J. Mass Spectrom. 2002, 221, 67-81.

33. Itina, T. E.; Zhigilei, L. V.; Garrison, B. J. Microscopic Mechanisms of Matrix Assisted Laser Desorption and Analyte Molecules: Insight from Molecular Dynamics Simulation. J. Phys. Chem. B 2002, 106, 303-310.

34. Spengler, B.; Kirsch, D. On the Formation of Initial Ion Velocities in Matrix-Assisted Laser Desorption ionization: Virtual Desorption Time as an Additional Parameter Describing Ion Ejection Dynamics. Int. J. Mass Spectrom. 2003, 226, 71-83.

35. Zhigilei, L. V.; Yingling, Y. G.; Itina, T. E.; Schoolcraft, T. A.; Garrison, B. J. Molecular Dynamics Simulation of MatrixAssisted Laser Desorption Connections to Experiment. Int. J. Mass Spectrom. 2003, 226, 85-106.

36. Gluckmann, M.; Karas, M. The Initial Ion Velocity and Its Dependence on Matrix, Analyte, and Preparation Method in Ultraviolet Matrix-Assisted Laser Desorption/Ionization. J. Mass Spectrom. 1999, 34, 467-477.

37. Karas, M.; Bahr, U.; Fournier, I.; Gluckmann, M.; Pfenninger, A. The Initial-Ion Velocity as a Marker for Different DesorptionIonization Mechanisms in MALDI. Int. J. Mass Spectrom. 2003, 226, 239-248.

38. Zhigilei, L. V.; Garrison, B. J. Velocity Distributions of Analyte Molecules in Matrix-Assisted Laser Desorption from Computer Simulations. Rapid Commun. Mass Spectrom. 1998, 12, 1273-1277.

39. Beavis, R. C.; Chait, B. T. Velocity Distributions of Intact High Mass Polypeptide Molecule Ions Produced by Matrix Assisted Laser Desorption. Chem. Phys. Lett. 1991, 181, 479-484.

40. Dreisewerd, K. The Desorption Process in MALDI. Chem. Rev. 2003, 103, 395-426.

41. Karas, M.; Gluckmann, M.; Schafer, J. Ionization in MatrixAssisted Laser Desorption/Ionization: Singly Charged Molecular Ions are the Luck Survivors. J. Mass Spectrom. 2000, 35, $1-12$.

42. Knochenmuss, R. A Quantitative Model of Ultraviolet MatrixAssisted Laser Desorption/Ionization. J. Mass Spectrom. 2002, 37, 867-877.

43. Zenobi, R.; Knochenmuss, R. Ion Formation in MALDI Mass Spectrometry. Mass Spectrom. Rev. 1998, 17, 337-366.

44. Berkenkamp, S.; Menzel, C.; Hillenkamp, F.; Dreisewerd, K. Measurements of Mean Initial Velocities of Analyte and Matrix Ions in Infrared Matrix-Assisted Laser Desorption Ionization Mass Spectrometry. J. Am. Soc. Mass Spectrom. 2002, 13, 209-220. 